\title{
S-duality and the double copy
}

\section{Rashid Alawadhi, David S. Berman, Bill Spence and David Peinador Veiga}

Centre for Research in String Theory, School of Physics and Astronomy, Queen Mary University of London, 327 Mile End Road, London E1 4NS, U.K.

E-mail: r.alawadhi@qmul.ac.uk, d.s.berman@qmul.ac.uk, w.j.spence@qmul.ac.uk, d.peinadorveiga@qmul.ac.uk

ABSTRACT: The double copy formalism provides an intriguing connection between gauge theories and gravity. It was first demonstrated in the perturbative context of scattering amplitudes but recently the formalism has been applied to exact classical solutions in gauge theories such as the monopole and instanton. In this paper we will investigate how duality symmetries in the gauge theory double copy to gravity and relate these to solution generating transformations and the action of $\operatorname{SL}(2, \mathbb{R})$ in general relativity.

Keywords: Classical Theories of Gravity, Duality in Gauge Field Theories, Scattering Amplitudes

ARXIV EPRINT: 1911.06797 


\section{Contents}

1 Introduction $\quad 1$

2 Classical double copy $\quad 3$

2.1 The Kerr-Schild double copy 3

2.2 Schwarzschild and NUT spacetimes 4

2.2.1 Schwarzschild spacetime 4

2.2.2 Taub-NUT spacetime 5

3 Solution generating transformations in general relativity $\quad 6$

3.1 Ehlers transformation 6

$\begin{array}{lll}3.2 & \text { Buchdahl's reciprocal transformation } & 8\end{array}$

4 The tensor Weyl double copy 10

$\begin{array}{ll}4.1 \text { Definitions } & 10\end{array}$

4.2 Ehlers and the double copy: the Schwarzschild case 11

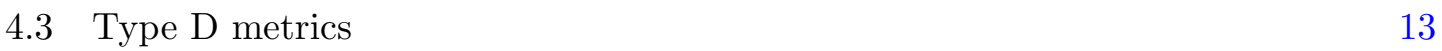

5 The $\operatorname{SL}(2, \mathbb{R})$ transformations $\quad 14$

$\begin{array}{lll}5.1 & \text { The spacetime Ehlers group } & 14\end{array}$

$\begin{array}{ll}5.2 \text { The Taub-NUT case } & 15\end{array}$

$\begin{array}{ll}5.3 \text { More general type D metrics } & 17\end{array}$

$\begin{array}{ll}5.4 \text { The Eguchi-Hanson metric } & 19\end{array}$

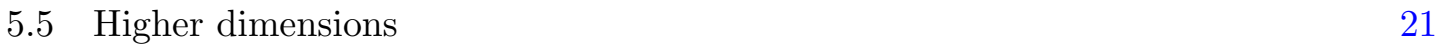

\section{Introduction}

The double copy was first investigated in a series of works [1-3] as a relationship between perturbative scattering amplitudes in gauge theory and gravity. It has been proved at tree level [3-11], where it has a stringy origin [12]. But there is still no non-perturbative proof of the double copy, although the evidence is mounting with a series of papers showing double copy behaviour for amplitudes at higher loop order [2, 13-43].

More recently, the double copy/single copy was applied to a class of exact classical solutions. Double copy refers to moving from gauge theory to gravity while single copy is the inverse map from gravity to gauge theory. The Schwarzschild solution was shown to single copy to an electric charge [44] and the Taub NUT solution single copy to a magnetic monopole [45]. Subsequent to that the single copy of the Eguchi-Hanson solution has been mapped to a self-dual gauge field [46]. More general topologically non-trivial solutions 
have been double copied in the work of [47]. (Other work examining symmetries of the linearised double copy have also appeared in a series of works [48-56].)

To futrher investigate the double copy formalism beyond the perturbative regime, we examine how non-perturbative symmetries in the gauge theory are double copied to gravity. In particular, gauge theories exhibit electromagnetic duality which exchanges electric and magnetic charges. This symmetry, often also called S-duality, was first discovered for classical Maxwell theory but then became a crucial ingredient in the study of properties of Yang-Mills theory [57]. General relativity is not known to exhibit S-duality which leads to the question of what S-duality could double copy to. We will address this at the non-perturbative classical level by identifying the solution generating symmetry in general relativity that single copies to electromagnetic duality.

We will show that the double copy of electromagnetic duality is identified as the Ehlers transformation in general relativity. It is worth commenting further that we are working with exact and thus classically non-perturbative solutions in the double copy and the metrics corresponding to the electric and magnetic solutions are mapped between each other using an exact non-local transformation. We will then demonstrate how another solution generating symmetry discovered by Buchdahl [58], is single copied to the charge conjugation.

We adopt two complementary approaches. First we will use the Kerr-Schild form of the metric where the metric in this form is related to gauge fields in the single copy. Second we will develop the correspondence introduced in [59] where the Weyl curvature in gravity is related to a combination of field strengths in the single copy. In each case we will examine how the electromagnetic transformation in the single copy is related to a transform in relativity.

To make the paper as self contained as possible and make clear our conventions for the double/single copy we begin with a description of the Kerr-Schild form in GR and then the single copy prescription. ${ }^{1}$ We give the detailed examples of the Schwarzschild black hole and the Taub-NUT solution and their single copies as described first in [44, 45]. Then we describe the Ehlers transform in general before moving on to its application to Schwarzchild and its single copy. After discussing the role of the the Buchdahl transform and its single copy again using Kerr-Schild form we move to the double copy in terms of curvatures and field strengths, known as the Weyl double copy [59] where we can again examine the role of electromagnetic duality from a complementary approach. We will show in a number of cases that the duality transformations in electromagnetism correspond with solution-generating transformations in general relativity, and together preserve the form of the double copy.

Note added. While this manuscript was in preparation [60] appeared which has overlap with this paper.

\footnotetext{
${ }^{1}$ Other conventions and methods for the double copy are also available, see for example [55, 56].
} 


\section{Classical double copy}

\subsection{The Kerr-Schild double copy}

First let use introduce the Kerr-Schild form of the metric and examine the behaviour of Einstein's field equations. Writing the metric in this form is a crucial step in making the double/single copy manifest. In what follows we shall use the procedure outlined in [44]. We take the metric $\eta_{\mu \nu}=\operatorname{diag}(-1,+1,+1,+1)$ throughout.

A solution is "Kerr-Schild" if a set of coordinates may be found such that the spacetime metric $g_{\mu \nu}$ may be put in the form

$$
g_{\mu \nu}=\eta_{\mu \nu}+\phi k_{\mu} k_{\nu}
$$

where $\phi$ is a scalar field and $k_{\mu}$ is a co-vector satisfying

$$
\eta^{\mu \nu} k_{\mu} k_{\nu}=0=g^{\mu \nu} k_{\mu} k_{\nu},
$$

i.e. it is null with respect to both the full and background metric. The inverse metric then takes the form

$$
g^{\mu \nu}=\eta^{\mu \nu}-\phi k^{\mu} k^{\nu} .
$$

In terms of the scalar field $\phi$ and co-vector $k_{\mu}$, the Ricci tensor and Ricci scalar are

$$
\begin{aligned}
R_{\nu}^{\mu} & =\frac{1}{2}\left(\partial^{\mu} \partial_{\alpha}\left(\phi k^{\alpha} k_{\nu}\right)+\partial_{\nu} \partial^{\alpha}\left(\phi k_{\alpha} k^{\mu}\right)-\partial^{2}\left(\phi k^{\mu} k_{\nu}\right)\right), \\
R & =\partial_{\mu} \partial_{\nu}\left(\phi k^{\mu} k^{\nu}\right),
\end{aligned}
$$

where $\partial^{\mu}=\eta^{\mu \nu} \partial_{\nu}$. In the stationary spacetime case $\left(\partial_{0} \phi=\partial_{0} k^{\mu}=0\right)$ one may take the time component of the Kerr-Schild vector as $k^{0}=1$, with the dynamics in the time component contained in $\phi$. As a consequence, the components of the Ricci tensor simplify to

$$
\begin{aligned}
R_{0}^{0} & =\frac{1}{2} \nabla^{2} \phi, \\
R_{0}^{i} & =-\frac{1}{2} \partial_{j}\left[\partial^{i}\left(\phi k^{j}\right)-\partial^{j}\left(\phi k^{i}\right)\right], \\
R_{j}^{i} & =\frac{1}{2} \partial_{l}\left[\partial^{i}\left(\phi k^{l} k_{j}\right)+\partial_{j}\left(\phi k^{l} k^{i}\right)-\partial^{l}\left(\phi k^{i} k_{j}\right)\right], \\
R & =\partial_{i} \partial_{j}\left(\phi k^{i} k^{j}\right),
\end{aligned}
$$

where Latin indices indicate the spatial components.

Now define a gauge field $A_{\mu}=\phi k_{\mu}$, with the Maxwell field strength $F_{\mu \nu}=\partial_{\mu} A_{\nu}-\partial_{\mu} A_{\nu}$. Taking the stationary case of the vacuum Einstein equations $R_{\mu \nu}=0$, one finds that the gauge field satisfies the Abelian Maxwell equations

$$
\partial_{\mu} F^{\mu \nu}=\partial_{\mu}\left(\partial^{\mu}\left(\phi k^{\nu}\right)-\partial^{\nu}\left(\phi k^{\mu}\right)\right)=0 .
$$

The remarkable thing about the double copy is that if now one considers a non-Abelian gauge field $A_{\mu}^{a}$ with the gauge group index $a$, there is still a single copy/double copy relationship. The recipe is to take the quantity $\phi k_{\mu} k_{\nu}$ of a given gravity solution and 
strip off one of the Kerr-Schild vectors and dress with a gauge group index to get the corresponding gauge field $A_{\mu}^{a}=c^{a} \phi k_{\mu}$. There is no derivation as such for this procedure but by now there is a compelling amount of evidence as listed in the introduction. Thus, the basic statement of the double/single copy we will be applying is:

If $g_{\mu \nu}=\eta_{\mu \nu}+\phi k_{\mu} k_{\nu}$ is a stationary solution of Einstein's equations, then $A_{\mu}^{a}=c^{a} \phi k_{\mu}$ is a solution of the Yang-Mills equations which is linearised by the Kerr-Schild coordinates allowing an arbitrary choice for the constant $c^{a}$.

\subsection{Schwarzschild and NUT spacetimes}

We shall demonstrate the double/single procedure on two important gravity solutions, the Schwarzschild black hole and the Taub NUT spacetime.

\subsubsection{Schwarzschild spacetime}

The Schwarzschild solution is

$$
d s^{2}=-\left(1-\frac{2 G M}{r}\right) d t^{2}+\frac{d r^{2}}{1-\frac{2 G M}{r}}+r^{2} d \Omega^{2},
$$

where $d \Omega^{2}=d \theta^{2}+\sin ^{2} \theta d \phi^{2}$ is the line element on the unit sphere, $G$ is Newton's constant, and $r^{2}=x^{2}+y^{2}+z^{2}$ is the radial distance from the origin.

In order to apply the single copy procedure, one must write this metric in Kerr-Schild form. To do this, we apply the coordinate transformation $l=t+\bar{r}$ with $d \bar{r}=\left(1-\frac{2 G M}{r}\right)^{-1} d r$, so that the metric takes the form

$$
d s^{2}=-d l^{2}+2 d l d r+r^{2} d \Omega^{2}+\frac{2 G M}{r} d l^{2} .
$$

A further coordinate transformation of the form $l=\bar{t}+r$ is then applied so that the metric becomes

$$
d s^{2}=-d \vec{t}^{2}+d r^{2}+r^{2} d \Omega^{2}+\frac{2 G M}{r}\left(d \vec{t}^{2}+d r^{2}+2 d \bar{t} d r\right) .
$$

Notice that the first three terms are just the flat Minkowski metric in spherical coordinates. Using the definition of $r$ given above to transform into 'Cartesian' coordinates the metric becomes

$$
d s^{2}=\eta_{\mu \nu} d x^{\mu} d x^{\nu}+\frac{2 G M}{r} k_{\mu} k_{\nu} d x^{\mu} d x^{\nu},
$$

where the null vector $k^{\mu}$ is defined by

$$
k^{\mu}=\left(1, \frac{x^{i}}{r}\right), \quad i=1 \ldots 3 .
$$

The metric is now in Kerr-Schild form:

$$
g_{\mu \nu}=\eta_{\mu \nu}+\frac{2 G M}{r} k_{\mu} k_{\nu} .
$$

In terms of a metric

$$
g_{\mu \nu}=\eta_{\mu \nu}+\kappa h_{\mu \nu}
$$


we have

$$
h_{\mu \nu}=\frac{\kappa}{2} \phi k_{\mu} k_{\nu}, \quad \phi=\frac{M}{4 \pi r} .
$$

The single copy then yields

$$
A^{\mu}=\frac{g c_{a} T^{a}}{4 \pi r} k^{\mu},
$$

where, motivated by the prescription for amplitudes, we have made the replacements

$$
\frac{\kappa}{2} \rightarrow g, \quad M \rightarrow c_{a} T^{a}, \quad k_{\mu} k_{\nu} \rightarrow k_{\mu} .
$$

The first one is just choosing the right coupling constant, the second one being the correspondence between the charges of the two theories.

\subsubsection{Taub-NUT spacetime}

The solution known as Taub-NUT was first derived by Taub [61] and generalised by Newman, Tamburino, and Unti [62] in 1963. The NUT solution is a non-asymptotically flat, axially symmetric stationary solution. Following Ortín [63], the Taub-NUT metric can be written as

$$
d s^{2}=-f(r)(d t-2 N \cos \theta d \phi)^{2}+f(r)^{-1} d r^{2}+\left(r^{2}+N^{2}\right) d \Omega_{(2)}^{2},
$$

where

$$
f(r)=\frac{\left(r-r_{+}\right)\left(r-r_{-}\right)}{r^{2}+N^{2}}, \quad r_{ \pm}=M \pm r_{0}, \quad r_{0}^{2}=M^{2}+N^{2} .
$$

This can be thought of as a generalisation of the Schwarzschild solution with an additional topological charge. This solution exhibits the following interesting properties:

- The solution is not trivial in the limit $M \rightarrow 0$.

- Taking the Newtonian limit shows that $M$ is indeed the mass of the source. The NUT charge has no Newtonian analogue.

- The solution defines its own class of asymptotic behaviour labeled by $N$ and is associated with the non-vanishing at infinity of the $g_{t \phi}$ component of the metric.

- The solution admits Dirac-like singularities at $\theta=0, \pi$, requiring the introduction of two coordinate patches.

We saw earlier that the mass in gravity single copies to an electric or colour charge. The NUT charge $N$ single copies to a magnetic monopole on the gauge theory side as follows.

The Taub-NUT metric written in coordinates introduced by Plebanski [64] exhibits a double Kerr-Schild form, as shown in [65]. This means the Taub-NUT metric may be written as:

$$
g_{\mu \nu}=\eta_{\mu \nu}+\kappa h_{\mu \nu}=\eta_{\mu \nu}+\kappa\left(\phi k_{\mu} k_{\nu}+\psi l_{\mu} l_{\nu}\right),
$$

where the vectors $k^{\mu}$ and $l^{\mu}$ must satisfy the following conditions

$$
k^{2}=l^{2}=k \cdot l=0, \quad(k \cdot D) k_{\mu}=0, \quad(l \cdot D) l_{\mu}=0 .
$$


This metric linearises the Einstein equations as with the single Kerr-Schild form. The generalisation of the single copy prescription for the gauge field is then

$$
A_{\mu}^{a}=c^{a}\left(\phi k_{\mu}+\psi l_{\mu}\right)
$$

Following [45], we have made the following substitutions

$$
\frac{M \kappa}{2} \rightarrow\left(c_{a} T^{a}\right) g_{s}, \quad \frac{N \kappa}{2} \rightarrow\left(c_{a} T^{a}\right) \tilde{g}_{s}
$$

The field strength is then

$$
F=\frac{1}{2} F_{\mu \nu} d x^{\mu} d x^{\nu}=-\frac{c_{a} T^{a}}{8 \pi}\left(\frac{g_{s}}{r^{2}} d t \wedge d r+\tilde{g}_{s} \sin \theta d \theta \wedge d \phi\right) .
$$

The first term on the right-hand side above is the pure electric charge corresponding to a Coulomb solution that was derived in the Schwarzschild case. The second term is a magnetic monopole charge which is single copied from the NUT contribution on the gravity side. In summary, the Schwarzschild single copies to a Coulomb solution and the NUT charge to a magnetic monopole.

\section{Solution generating transformations in general relativity}

A solution generating transformation is simply a recipe for obtaining a new solution to Einstein's equations from a known one. Their existence is remarkable given that Einstein's equations are a set of ten second order coupled non-linear differential equations that are notoriously difficult to solve. Whilst explicit symmetries in Einstein's equations make some transformations straightforward, there are "hidden symmetries" that allow us to generate very non-trivial solutions through this transformation technique. This approach to solving Einstein's equations was studied by Buchdahl, Ehlers, Geroch and Ernst [66]. The transformations require a Killing symmetry in the spacetime. When such a symmetry is present then there are a set of solution-generating techniques which we describe below.

\subsection{Ehlers transformation}

The Ehlers transformation is a transformation that acts on the parameters of a static solution of Einstein gravitational field equations and generates other solutions of the field equations that are in general stationary. In what follows we describe how the Ehlers transformation works [67], following [68]. It is assumed that the spacetime possesses a timelike Killing vector $\xi$ that generates an isometry. First perform a $(1+3)$ decomposition of the metric and choose coordinates $x^{\mu}=\left\{x^{0}, x^{i}\right\}$ to put the line element in the following form

$$
d s^{2}=-e^{2 U}\left(d x^{0}+A_{i} d x^{i}\right)^{2}+d \ell^{2},
$$

where we define

$$
A_{i}=\frac{-g_{0 i}}{g_{00}}, \quad e^{2 U}=-g_{00}, \quad d l^{2}=\gamma_{i j} d x^{i} d x^{j}
$$


and

$$
\gamma_{\alpha \beta}=\left(-g_{\alpha \beta}+\frac{g_{0 \alpha} g_{0 \beta}}{g_{00}}\right) .
$$

The Ehlers transformation states that if

$$
g_{\mu \nu} d x^{\mu} d x^{\nu}=-e^{2 U}\left(d x^{0}\right)^{2}+e^{-2 U} d \tilde{\ell}^{2},
$$

with $d \tilde{\ell}^{2}=e^{2 U} d \ell^{2}$, is the metric of a static spacetime, then

$$
\bar{g}_{\mu \nu} d x^{\mu} d x^{\nu}=-(\alpha \cosh (2 U))^{-1}\left(d x^{0}+A_{i} d x^{i}\right)^{2}-\alpha \cosh (2 U) d \tilde{\ell}^{2},
$$

where $\alpha$ is a positive constant, $U=U\left(x^{i}\right)$ and $A_{i}=A_{i}\left(x^{j}\right)$, is the metric of a stationary spacetime provided that $A_{i}$ satisfies Ehlers equation

$$
-\alpha \sqrt{\tilde{\gamma}} \epsilon_{i j k} U^{, k}=A_{[i, j]},
$$

where $\tilde{\gamma}_{a b}$ is the conformal spatial metric. Using this method, one generates a stationary solution from a static one by finding the potential $A_{a}$ which is related to a given static potential $U$. Let us see how this comes about by applying this procedure to the Schwarzschild metric.

Start with the Schwarzschild metric in the form (3.4), with the potential taking the form

$$
U_{c}=\frac{1}{2} \ln \left(1-\frac{2 m}{r}\right)+C
$$

and

$$
d \tilde{\ell}^{2}=d r^{2}+r^{2} e^{2 U}\left(d \theta^{2}+\sin ^{2} \theta d \phi^{2}\right),
$$

where $C$ is a constant and $U\left(x^{a}\right)$ only depends on the spatial coordinates. Then the Ehlers equation (3.6) reads

$$
-\alpha r^{2} e^{2 U} \sin \theta \tilde{\gamma}^{r r} U_{, r}=\frac{1}{2}\left(A_{\theta, \phi}-A_{\phi, \theta}\right) .
$$

One may choose a gauge such that $A_{\theta, \phi}=0$. Then substituting (3.7) in the above equation and solving for the field $A_{\phi}$ we find

$$
A_{\phi}(\theta)=-2 \alpha m \cos \theta \text {. }
$$

The resulting metric is then given by (3.5) as

$$
d s^{2}=-\frac{r(r-2 m)}{\alpha f(r)}(d t-2 \alpha m \cos \theta d \phi)^{2}+\frac{\alpha f(r)}{r(r-2 m)} d r^{2}+\alpha f(r) d \Omega^{2},
$$

where

$$
f(r)=r^{2}\left(\frac{1+c_{1}^{2}}{2 c_{1}}\right)+2 m^{2} c_{1}-2 m r c_{1}, \quad c_{1}=e^{2 C} .
$$

Recovering the Schwarzschild metric as $\alpha \rightarrow 0$ requires us to set

$$
\alpha=\frac{2 c_{1}}{1+c_{1}^{2}}, \quad\left|c_{1}\right| \leq 1 .
$$


Now apply the following changes of variables and redefinitions of constants;

$$
M=m \frac{1-c_{1}^{2}}{1+c_{1}^{2}}, \quad \alpha m=N, \quad r-N c_{1}=R,
$$

to get

$$
d s^{2}=-\frac{R^{2}-2 M R-N^{2}}{R^{2}+N^{2}}(d t-2 N \cos \theta d \phi)^{2}+\frac{R^{2}+N^{2}}{R^{2}-2 M R-N^{2}} d R^{2}+\left(R^{2}+N^{2}\right) d \Omega^{2},
$$

which is the metric for the NUT spacetime with NUT charge $N$. Remarkably, the constant $C$ in the potential $U$, while having no effect on the Schwarzschild spacetime, plays an important role in generating solutions when the Ehlers transformation is applied. Had we started with $C=0$ we would have ended up with the pure NUT spacetime $(M=0)$. This means that the seed metric for both the NUT with mass and pure NUT metric is the Schwarzschild metric.

\subsection{Buchdahl's reciprocal transformation}

In [58] Buchdahl showed that if a solution of Einstein's equations admits a Killing vector field one can obtain a new solution by applying the so called "reciprocal transformation". For coordinates adapted to the Killing direction such that the Killing vector is given by $\frac{\partial}{\partial x^{\alpha}}$, then the line element may be written

$$
d s^{2}=g_{\beta \gamma} d x^{\beta} d x^{\gamma}+g_{\alpha \alpha}\left(d x^{\alpha}\right)^{2} .
$$

The reciprocal transformation then generates the following line element

$$
d s^{2}=g_{\alpha \alpha}^{\frac{2}{d-3}} g_{\beta \gamma} d x^{\beta} d x^{\gamma}+g_{\alpha \alpha}^{-1}\left(d x^{\alpha}\right)^{2} .
$$

Thus transformation may be written acting on metric components in this adapted coordinate system as

$$
\left(g_{\alpha \alpha}, g_{\beta \gamma}\right) \rightarrow\left(g_{\alpha \alpha}^{-1}, g_{\alpha \alpha}^{\frac{2}{d-3}} g_{\beta \gamma}\right) .
$$

The reader familiar with string theory will note that Buchdahl's reciprocal transformation is essentially T-duality as a solution generating transformation, predating it by some thirty years. We shall apply this to the Schwarzschild metric. As noted above, the $d=4$ Schwarzschild metric admits a time-like Killing vector $\partial / \partial t$ so that the transformation yields

$$
\left(g_{t t}, g_{i j}\right) \rightarrow\left(g_{t t}^{-1}, g_{t t}^{2} g_{i j}\right),
$$

which upon substitution into the metric gives

$$
d s^{2}=-\frac{d t^{2}}{1-\frac{2 M}{r}}+\left(1-\frac{2 M}{r}\right) d r^{2}+r^{2}\left(1-\frac{2 M}{r}\right)^{2}\left(d \theta^{2}+\sin \theta^{2} d \phi^{2}\right) .
$$

The Schwarzschild metric also admits the spatial Killing vector $\partial / \partial \phi$. Applying the same transformation, but now with $\alpha=\phi$, we get the following metric

$$
d s^{2}=-r^{4} \sin ^{4} \theta\left(1-\frac{2 M}{r}\right) d t^{2}+r^{4} \sin ^{4} \theta\left(1-\frac{2 M}{r}\right)^{-1} d r^{2}+r^{6} \sin ^{4} \theta d \theta^{2}+\frac{1}{r^{2} \sin ^{2} \theta} d \phi^{2} .
$$


This new solution is completely unrelated to the original seed metric, unlike (3.20) which was obtained using the time-like Killing symmetry. However, the metric (3.20) is related to the Schwarzschild metric by a simple coordinate transformation $R=r-2 M$ whereby one obtains the Schwarzschild metric but with negative mass parameter

$$
d s^{2}=-\left(1-\frac{2 M}{R}\right) d t^{2}+\left(1+\frac{2 M}{R}\right)^{-1} d R^{2}+R^{2}\left(d \theta^{2}+\sin \theta^{2} d \phi^{2}\right) .
$$

If we write the negative mass Schwarzschild metric in in Kerr-Schild form

$$
g_{\mu \nu}=\eta_{\mu \nu}-\frac{2 G M}{r} k_{\mu} k_{\nu}
$$

and then do the usual single copy procedure where $-M \rightarrow-c_{a} T^{a}$ we arrive at

$$
\tilde{A}_{\mu}=\left(-\frac{g c_{a} T^{a}}{4 \pi r}, 0,0,0\right),
$$

which has the opposite sign of the gauge field $A_{\mu}$ compared to the single copy of the positive mass Schwarzschild solution [44], i.e.

$$
\text { 'Single copied 4D Buchdahl' : } A_{\mu} \rightarrow-A_{\mu} \text {. }
$$

This shows that the Buchdahl reciprocal transformation associated with the time-like Killing vector in the Schwarzschild spacetime is the gravitational analogue of charge conjugation on the gauge theory side.

We now examine this transformation acting on the Taub-NUT solution by first using the Schwarzschild metric as our seed metric on which we act with the Buchdahl transform, followed by the Ehler transformation. Firstly, note that Buchdahl transformation corresponds to sending $U \rightarrow \hat{U}=-U$ when one writes the Schwarzschild metric in the form

$$
d s^{2}=-e^{2 U}\left(d x^{0}\right)^{2}+e^{-2 U} d \tilde{\ell}^{2}, \quad U=\frac{1}{2} \ln \left(1-\frac{2 M}{r}\right) .
$$

Thus the Buchdahl-transformed Schwarzschild metric reads

$$
d s^{2}=-e^{-2 U}\left(d x^{0}\right)^{2}+e^{2 U} d \hat{\ell}^{2}, \quad d \hat{\ell}^{2}=d r^{2}+e^{-2 U} r^{2} d \Omega^{2} .
$$

which as before is just the Schwarzschild metric with negative mass as shown earlier. Now construct the Ehlers transformed metric as before. Considering the Ehlers equation for the reciprocal solution

$$
-\alpha \sqrt{\gamma} \epsilon_{\alpha \beta \gamma} \hat{U}^{, \gamma}=\alpha \sqrt{\gamma} \epsilon_{\alpha \beta \gamma} U^{, \gamma}=\hat{A}_{[\alpha, \beta]}
$$

and comparing it to the standard one (3.6) we find

$$
\hat{A}_{\mu}=-A_{\mu} \text {. }
$$

Then, following exactly the same steps as before, i.e. solving for $A_{\mu}$ while imposing axial symmetry, we find

$$
\hat{A}_{\phi}(\theta)=2 \alpha M \cos \theta
$$


(up to a constant), and as before the NUT charge is given by

$$
\hat{N}=\alpha M=-N \text {. }
$$

This has acquired a negative sign, comparing the field $A_{\mu}$ to eq. (3.10). The reciprocal Taub-NUT metric then reads

$$
\begin{aligned}
& d s^{2}=-(\alpha \cosh (2 U))^{-1}\left(d x^{0}+\hat{A}_{\beta} d x^{\beta}\right)^{2}+\alpha \cosh (2 U) d \hat{\ell}^{2}, \\
& d \hat{\ell}^{2}=d r^{2}+e^{-2 U} r^{2} d \Omega^{2} .
\end{aligned}
$$

Upon applying the single copy procedure [45] as in the previous sections using the multiKerr-Schild form of the metric one finds again that the resulting gauge field has negative electric and magnetic monopole charges.

In summary, we show below diagrams of the transformations and their effects on the parameters of both the Schwarzschild and Taub-NUT solutions. The vertical lines indicate the application of the double/single copy ("S.D.C") procedure.
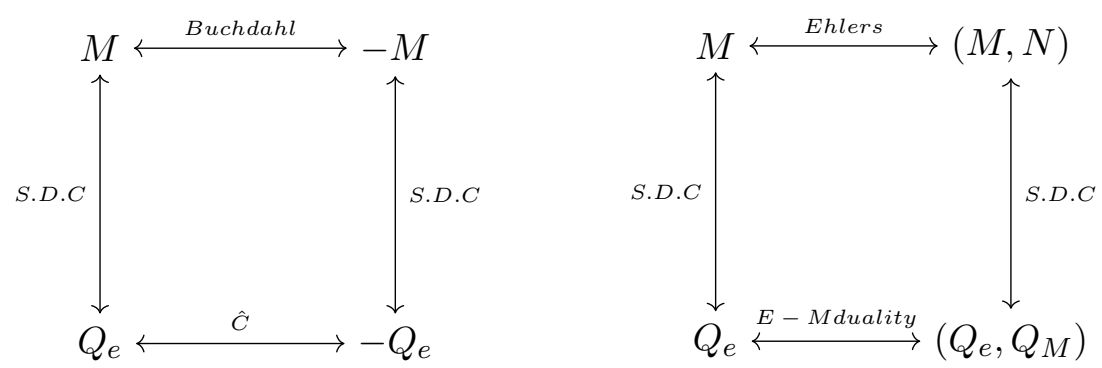

From the action of the Ehlers transformation on the charges in the double copy one sees that the single copy of the Ehlers transformation is electromagnetic duality in the gauge theory; alternatively the double copy of electromagnetic duality is generated by the Ehlers transformation in gravity.

More formally, the Ehlers transformation is an element of $\operatorname{SL}(2, \mathbb{R})$ as introduced by Geroch [69]. However when this acts on the Taub NUT solution the quantisation of NUT charge means that the group must be broken to $\mathrm{SL}(2, \mathbb{Z})$ so as to preserve the quantisation condition. This exactly follows what happens with the electromagnetic duality group acting on the dyon spectrum. Classically the group is $\operatorname{SL}(2, \mathbb{R})$ but this reduces to $\mathrm{SL}(2, \mathbb{Z})$ in order to maintain Dirac quantisation for the magnetic charges.

\section{The tensor Weyl double copy}

\subsection{Definitions}

The double copy as described above is defined in terms of the gauge connection and the metric. It is a natural question to ask whether one might study a double copy directly in terms of field strengths and curvatures. This was investigated in [59], where a particularly nice form of the double copy was obtained using spinors - writing the spinor corresponding to the Weyl tensor in terms of the spinor for the Maxwell tensor as

$$
C_{A B C D}=\frac{1}{S} f_{(A B} f_{C D)}
$$


where $S$ is a suitable function related to the "zeroth copy". This "Weyl double copy" was shown to be consistent with the previously known Kerr-Schild double copy, and resolved some of ambiguities in that formulation. It also presented new double copy interpretations of the Eguchi-Hanson instanton, and the C-metric, relating the latter to the Liénard-Wiechert potential for a pair of uniformly accelerated charges. Extending this four-dimensional result to higher dimensions requires an appropriate study of spinors and curvature invariants in higher dimensions, and the latter has been explored recently in [70].

A higher-dimensional Weyl double copy might also be investigated in terms of a tensor version of the spinor Weyl double copy. One can obtain this by translation from the spinor equations of course; more directly one can note that this must involve writing the Weyl tensor as a sum of terms quadratic in the Maxwell tensor. Keeping in mind the algebraic symmetries of the Weyl tensor, in four dimensions there are two independent expressions that one can write down:

$$
\begin{aligned}
& C_{\mu \nu \rho \sigma}[F]=F_{\mu \nu} F_{\rho \sigma}-F_{\rho \mu} F_{\nu \sigma}-3 g_{\mu \rho} F_{\nu}{ }^{\lambda} F_{\sigma \lambda}+\left.\frac{1}{2} g_{\mu \rho} g_{\nu \sigma} F^{2}\right|_{s}, \\
& D_{\mu \nu \rho \sigma}[F]=\frac{1}{2}\left(F_{\mu \nu} \tilde{F}_{\rho \sigma}-F_{\rho \mu} \tilde{F}_{\nu \sigma}-3 g_{\mu \rho} F_{\nu}{ }^{\lambda} \tilde{F}_{\sigma \lambda}+\frac{1}{2} g_{\mu \rho} g_{\nu \sigma} F . \tilde{F}\right)+\left.(F \leftrightarrow \tilde{F})\right|_{s},
\end{aligned}
$$

where $F^{2}=F^{\lambda \delta} F_{\lambda \delta}, F \cdot \tilde{F}=F^{\lambda \delta} \tilde{F}_{\lambda \delta}$, and $\tilde{F}_{\mu \nu}=\frac{1}{2} \sqrt{g} \epsilon_{\mu \nu \rho \sigma} F^{\rho \sigma}$ with $\epsilon_{\mu \nu \rho \sigma}$ the numerical alternating symbol. In equations like those above, the symbol " $s_{s}$ " applies to the expression on the right-hand side of the equation, and it means to anti-symmetrise in the indices $\mu, \nu$ and in $\rho, \sigma$, with unit weight. In $D$ dimensions there is no equivalent of $D_{\mu \nu \rho \sigma}[F]$ and one just has

$$
C_{\mu \nu \rho \sigma}^{(D)}[F]=F_{\mu \nu} F_{\rho \sigma}-F_{\rho \mu} F_{\nu \sigma}-\frac{6}{D-2} g_{\mu \rho} F_{\nu}{ }^{\lambda} F_{\sigma \lambda}+\left.\frac{3}{(D-1)(D-2)} g_{\mu \rho} g_{\nu \sigma} F^{2}\right|_{s} .
$$

We make some comments on the higher-dimensional double copy at the end of the paper and hereon consider four dimensions. A four-dimensional Weyl tensor double copy must involve a linear sum of the two expressions $C_{\mu \nu \rho \sigma}$ and $D_{\mu \nu \rho \sigma}$, with suitable coefficients which will in general be functions of the relevant variables and constants. We note some useful properties of these expressions: $C_{\mu \nu \rho \sigma}[\tilde{F}]=C_{\mu \nu \rho \sigma}[F], \tilde{C}_{\mu \nu \rho \sigma}[F]=D_{\mu \nu \rho \sigma}[F], D_{\mu \nu \rho \sigma}[\tilde{F}]=-D_{\mu \nu \rho \sigma}[F]$ and

$$
C_{\mu \nu \rho \sigma}[a F+b \tilde{F}]=\left(a^{2}+b^{2}\right) C_{\mu \nu \rho \sigma}[F]+2 a b D_{\mu \nu \rho \sigma}[F]
$$

for any coefficients $a, b$. Define the self-dual and anti-self-dual parts of a two form $F$ via $F^{ \pm}=\frac{1}{2}(F \pm \tilde{F})$ with a similar formula for $C^{ \pm}$, with the action either on the left or right pair of indices. Then

$$
C_{\mu \nu \rho \sigma}^{ \pm}[a F+b \tilde{F}]=C_{\mu \nu \rho \sigma}\left[(a \pm b) F^{ \pm}\right]
$$

\subsection{Ehlers and the double copy: the Schwarzschild case}

We would like to explore how the Ehlers transformation described earlier may be understood in terms of the double copy. Consider as starting point the Schwarzschild metric. 
We will find it useful to work in coordinates $(u, v, p, q)$ with the metric

$$
\begin{aligned}
d s^{2}= & \frac{1}{(1-p q)^{2}}\left[2 i\left(d u+q^{2} d v\right) d p-2\left(d u-p^{2} d v\right) d q+\frac{2 m p^{3}}{\left(p^{2}+q^{2}\right)}\left(d u+q^{2} d v\right)^{2}\right. \\
& \left.+\frac{2 m q}{\left(p^{2}+q^{2}\right)}\left(d u-p^{2} d v\right)^{2}\right] .
\end{aligned}
$$

The single copy Maxwell tensor is then [59]

$$
F_{S}=\frac{e}{\left(p^{2}+q^{2}\right)^{2}}\left[2 p q\left(d u+q^{2} d v\right) d p-\left(p^{2}-q^{2}\right)\left(d u-p^{2} d v\right) d q\right] .
$$

It is then straightforward to show that the Weyl tensor $C_{S}$ for the Schwarzschild metric is given by

$$
C_{\mu \nu \rho \sigma}^{S}=-\left.\frac{4}{(1-p q) e}\left(q C_{\mu \nu \rho \sigma}\left[F_{S}\right]-i p D_{\mu \nu \rho \sigma}\left[F_{S}\right]\right)\right|_{e \rightarrow m}
$$

where $\left.\right|_{e \rightarrow m}$ means to replace $e$ by $m$ on the right-hand side of the equation. This result may be written simply as

$$
C_{\mu \nu \rho \sigma}^{S}=-\left.\frac{4}{(1-p q)}\left(C_{\mu \nu \rho \sigma}\left[\alpha_{S} F_{S}^{+}+(c . c)\right]\right)\right|_{e \rightarrow m}
$$

where $\alpha_{S}=\sqrt{\frac{-i(p+i q)}{e}}$.

Now let us consider the Taub-NUT metric in the corresponding coordinate system:

$$
\begin{aligned}
d s^{2}= & \frac{1}{(1-p q)^{2}}\left[2 i\left(d u+q^{2} d v\right) d p-2\left(d u-p^{2} d v\right) d q+2 p \frac{m p^{2}+n}{\left(p^{2}+q^{2}\right)}\left(d u+q^{2} d v\right)^{2}\right. \\
& \left.+2 q \frac{m+n q^{2}}{\left(p^{2}+q^{2}\right)}\left(d u-p^{2} d v\right)^{2}\right]
\end{aligned}
$$

where $n$ is the Taub-NUT charge. The single copy Maxwell tensor in this case is

$$
\left.F_{T}=\frac{1}{\left(p^{2}+q^{2}\right)^{2}}\left[\left(2 e p q+g\left(p^{2}-q^{2}\right)\right)\left(d u+q^{2} d v\right) d p+\left(2 g p q-e\left(p^{2}-q^{2}\right)\right)\left(d u-p^{2} d v\right) d q\right)\right] .
$$

This can be expressed simply in terms of the Schwarzschild Maxwell single copy tensor as

$$
F_{T}=F_{S}-\frac{i g}{e} \tilde{F}_{S}
$$

Now, if we make the replacements

$$
F_{S} \rightarrow F_{S}-\frac{i g}{e} \tilde{F}_{S}, \quad e \rightarrow e-i g
$$

on the right-hand side of (4.10), and then make the replacements $e \rightarrow m, g \rightarrow n$ then we find a tensor that we will call $C^{T}$ which is

$$
C_{\mu \nu \rho \sigma}^{T}:=-\left.\frac{4}{(1-p q)}\left(C_{\mu \nu \rho \sigma}\left[\alpha_{T} F_{T}^{+}+(c . c)\right]\right)\right|_{e \rightarrow m, g \rightarrow n}
$$


where $\alpha_{T}=\sqrt{\frac{-i(p+i q)}{(e-i g)}}$. It can be checked that $C^{T}$ is the Weyl tensor for the Taub-NUT metric (4.11). (Note that we implicitly assumed that the charge $e$ is complex prior to the shift, and that in going from (4.10) to (4.15) the metric dependence in $C_{\mu \nu \rho \sigma}[F]$ also needs to shift from (4.7) to (4.11).)

Thus we see that the Ehlers transformation which takes one from the Schwarzschild to the Taub-NUT spacetime can be seen via the Weyl double copy as a simple duality transformation (4.14) (combined with identifying $(e, g)$ with $(m, n)$ ) which maps between the two Weyl double copy curvatures. It is instructive to return to the spinor form of the Weyl double copy (4.1) in the light of this (see section 4 of [59]). The transformation $e F \rightarrow(e-i g) F$ induces the shifts $e F^{ \pm} \rightarrow(e \mp i g) F^{ \pm}$. The Maxwell field strength spinor $f_{A B}$ depends only on the self-dual part of the Maxwell tensor and thus transforms according to this formula. The scale function $e S$ in (4.1) transforms to $(e-i g) S$ and hence the double copy formula yields

$$
m C_{A B C D} \rightarrow(m-i n) C_{A B C D}
$$

correctly mapping the Schwarzschild Weyl spinor to the Taub-NUT one.

\subsection{Type D metrics}

The Taub-NUT example considered above is a special case of the general vacuum type D solution with vanishing cosmological constant [71], as given in [59]:

$d s^{2}=\frac{1}{(1-p q)^{2}}\left[2 i\left(d u+q^{2} d v\right) d p-2\left(d u-p^{2} d v\right) d q+\frac{P(p)}{p^{2}+q^{2}}\left(d u+q^{2} d v\right)^{2}-\frac{Q(q)}{p^{2}+q^{2}}\left(d u-p^{2} d v\right)^{2}\right]$,

with

$$
\begin{aligned}
& P(p)=\gamma\left(1-p^{4}\right)+2 n p-\epsilon p^{2}+2 m p^{3}, \\
& Q(q)=\gamma\left(1-q^{4}\right)-2 m q+\epsilon q^{2}-2 n q^{3},
\end{aligned}
$$

where the parameters $m, n, \gamma, \epsilon$ are related to the mass, NUT charge, angular momentum and acceleration (see [72] for a discussion of the various limits and definitions which enable the identifications in different cases).

The single copy Maxwell tensor in this case is the same as the one for the Taub-NUT metric (4.11). It is then natural to investigate the Weyl double copy in this case and, indeed, one finds that the Weyl tensor $C^{D}$ for the type D metric (4.17) is given by the same formula as that for the TN case:

$$
C_{\mu \nu \rho \sigma}^{D}:=-\left.\frac{4}{(1-p q)}\left(C_{\mu \nu \rho \sigma}\left[\alpha_{T} F_{T}^{+}+(c . c)\right]\right)\right|_{e \rightarrow m, g \rightarrow n},
$$

with $\alpha_{T}=\sqrt{\frac{-i(p+i q)}{(e-i g)}}$. Note that the metric (4.17) enters the right-hand side of (4.19) so that this doesn't simply reproduce $C_{\mu \nu \rho \sigma}^{T}$.

One can then ask if an Ehlers transformation will take one from the spacetime with Type D metric (4.17) with $n=0$, to that with nonzero $n$. To see this, consider the type $\mathrm{D}$ 
metric $g_{D_{0}}$ with vanishing NUT charge. This satisfies

$$
C_{\mu \nu \rho \sigma}^{D_{0}}=-\left.\frac{4}{(1-p q)}\left(C_{\mu \nu \rho \sigma}\left[\alpha_{S} F_{S}^{+}+(c . c)\right]\right)\right|_{e \rightarrow m} .
$$

Then if we make the replacements $F_{S} \rightarrow F_{S}-\frac{i g}{e} \tilde{F}_{S}$ and $e \rightarrow e-i g$ in the right-hand side of the above, and shift the metric from $g_{D_{0}}$ to (4.17), we find that we reproduce (4.19).

\section{The $\mathrm{SL}(2, \mathbb{R})$ transformations}

\subsection{The spacetime Ehlers group}

We would now like to discuss how $\operatorname{SL}(2, \mathbb{R})$ transformations act more generally in the context of the double copy. For this purpose, it will be useful to use a generalisation of the Ehlers procedure for more general Killing vector fields: the spacetime Ehlers group [73], which we now summarise briefly. ${ }^{2}$ Given a Killing vector field $\xi=\xi^{\mu} \partial_{\mu}$ and one-form $W=W_{\mu} d x^{\mu}$ on a Lorentzian manifold with metric $g_{\mu \nu}$, satisfying the vacuum Einstein equations, the spacetime Ehlers group is defined in [73] by the transformation

$$
g_{\mu \nu} \rightarrow \Omega^{2} g_{\mu \nu}-2 \xi_{(\mu} W_{\nu)}-\frac{\lambda}{\Omega^{2}} W_{\mu} W_{\nu}
$$

where $\lambda=-\xi^{\mu} \xi_{\mu}$ and $\Omega^{2} \equiv \xi^{\mu} W_{\mu}+1 \geq 1$ with the inequality holding over the whole geometry. Define the twist potential $\omega_{\mu}=\sqrt{-\operatorname{det}(g)} \epsilon_{\mu \nu \sigma \rho} \xi^{\nu} \nabla^{\sigma} \xi^{\rho}$ and the Killing tensor two form $F_{\mu \nu}=2 \partial_{[\mu} \xi_{\nu]}$ (note that we have a factor of 2 here, and a factor of $1 / 2$ in the definitions of the (anti-)self-dual parts of $F$, in comparison with [73]). Then the Ernst one-form

$$
\sigma_{\mu}:=2 \xi^{\nu} F_{\nu \mu}^{+}=\nabla_{\mu} \lambda-i \omega_{\mu}
$$

is closed, following from the vanishing of the Ricci tensor, and so locally $\sigma_{\mu}=\nabla_{\mu} \sigma$ for some complex function $\sigma .^{3}$

The spacetime Ehlers group is then defined for $W$ satisfying

$$
\begin{aligned}
2 \nabla_{[\mu} W_{\nu]} & =-4 \gamma \operatorname{Re}\left[(\gamma \bar{\sigma}+i \delta) F_{\mu \nu}^{+}\right], \\
\xi^{\mu} W_{\mu}+1 & =(i \gamma \sigma+\delta)(-i \gamma \bar{\sigma}+\delta),
\end{aligned}
$$

where a bar denotes complex conjugation, and $\gamma$ and $\delta$ are non-simultaneously vanishing real constants, which as a pair fix the gauge of $W$. After repeated action, the transformation defines an $\mathrm{SL}(2, \mathbb{R})$ group action on the Ernst scalar by the Möbius map

$$
\sigma \rightarrow \frac{\alpha \sigma+i \beta}{i \gamma \sigma+\delta}, \quad \text { where } \quad \beta \gamma+\alpha \delta=1
$$

\footnotetext{
${ }^{2}$ We comment that this paper and work following from it (see for example [74] and references therein) anticipate some of the formulæ of the double copy - e.g., the vanishing of the Mars-Simons tensor defined below corresponds to the self-dual part of the tensor double copy, and the spinor form of this can be found in $[75]$.

${ }^{3}$ This satisfies $\nabla^{2} \sigma=-\left(F_{\mu \nu}^{+}\right)^{2}$. In this construction we do not see a direct emergence of the standard harmonic "zeroth copy" function, as in the Kerr-Schild formulation.
} 
The self-dual part of the Killing tensor transforms as

$$
F_{\mu \nu}^{+} \rightarrow \frac{1}{(i \gamma \sigma+\delta)^{2}}\left(\Omega^{2} F_{\mu \nu}^{+}-W_{[\mu} \sigma_{\nu]}\right)
$$

where $W, \sigma$ are the one-forms defined above. The self-dual part of the Weyl tensor transforms as

$$
C_{\mu \nu \rho \sigma}^{+} \rightarrow \frac{1}{(i \gamma \sigma+\delta)^{2}} P_{\mu \nu}^{\alpha \beta} P_{\rho \sigma}^{\gamma \delta}\left(C_{\alpha \beta \gamma \delta}^{+}-\frac{6 i \gamma}{i \gamma \sigma+\delta}\left(F_{\alpha \beta}^{+} F_{\gamma \delta}^{+}-\frac{1}{3} I_{\alpha \beta \gamma \delta}\left(F^{+}\right)^{2}\right)\right),
$$

where in our conventions $I_{\mu \nu \rho \sigma}=\frac{1}{4}\left(g_{\mu \rho} g_{\nu \sigma}-g_{\nu \rho} g_{\mu \sigma}+\epsilon_{\mu \nu \rho \sigma}\right)$ is the canonical metric in the space of self-dual two-forms and $P_{\mu \nu}^{\alpha \beta}=\Omega^{2} \delta_{\mu}^{\alpha} \delta_{\nu}^{\beta}-\delta_{\mu}^{\alpha} \xi^{\beta} W_{\nu}-\xi^{\alpha} W_{\mu} \delta_{\nu}^{\beta}$. Notice that

$$
F_{\mu \nu}^{+} F_{\rho \sigma}^{+}-\frac{1}{3} I_{\mu \nu \rho \sigma}\left(F^{+}\right)^{2}=\frac{2}{3} C_{\mu \nu \rho \sigma}\left[F^{+}\right]
$$

in terms of the definition in (4.2). The Mars-Simons tensor is then defined as

$$
S_{\mu \nu \rho \sigma}=C_{\mu \nu \rho \sigma}^{+}-\frac{2}{3} Q C_{\mu \nu \rho \sigma}\left[F^{+}\right]
$$

for a suitable function $Q$. Finally, as discussed in section 6 of [73], we note that the vanishing of the Mars-Simons tensor is maintained under the Ehlers transformation, with $Q$ transforming appropriately.

\subsection{The Taub-NUT case}

Let us now consider applying these arguments in the context of the Weyl double copy described earlier. Starting from the Taub-NUT metric, in the real form (2.20), consider the Killing vector $\xi=\partial_{t}$. Its associated two-form $F_{\mu \nu}=2 \partial_{[\mu} \xi_{\nu]}$ is

$$
\begin{aligned}
F= & \frac{2 M\left(r^{2}-N^{2}\right)+4 N^{2} r}{\left(N^{2}+r^{2}\right)^{2}} d t \wedge d r+\frac{4 N \cos (\theta)\left(M\left(r^{2}-N^{2}\right)+2 N^{2} r\right)}{\left(N^{2}+r^{2}\right)^{2}} d r \wedge d \phi \\
& +\frac{2 N \sin (\theta)\left(r(2 M-r)+N^{2}\right)}{N^{2}+r^{2}} d \theta \wedge d \phi .
\end{aligned}
$$

This solves the Maxwell equations on the Taub-NUT background. The single copy of TaubNUT was found in $[45,59]$ and solves the flat-background Maxwell equations. The Ernst one-form is obtained from its definition

$$
\sigma_{\mu}:=2 \xi^{\nu} F_{\nu \mu}^{+}=\frac{2(M-i n)}{(r-i n)^{2}} \delta_{\mu}^{r} .
$$

In [73] it was proved that the Ernst one-form is exact, $\sigma_{\mu}=\partial_{\mu} \sigma$, and the integration constant can be chosen such that $\operatorname{Re}(\sigma)=-\xi^{\mu} \xi_{\mu}$, giving

$$
\sigma=1-\frac{2(N+i M)}{N+i r}
$$


Additionally, the fact that (2.20) has a Weyl double copy structure implies that

$$
\begin{aligned}
C_{\alpha \beta \gamma \delta}^{+} & =-\frac{6}{c-\sigma}\left(F_{\alpha \beta}^{+} F_{\gamma \delta}^{+}-\frac{\left(F^{+}\right)^{2}}{3} I_{\alpha \beta \gamma \delta}\right), \\
\left(F^{+}\right)^{2} & =A(c-\sigma)^{4}
\end{aligned}
$$

with $c=1$ and $A=-(4(M-i N))^{-1}$. Next, $W$ is found by solving (5.3). After this, we can transform the original metric into (5.1)

$$
g_{\mu \nu}^{\prime}=\Omega^{2} g_{\mu \nu}-2 \xi_{(\mu} W_{\nu)}+\frac{\xi^{\sigma} \xi_{\sigma}}{\Omega^{2}} W_{\mu} W_{\nu}
$$

In order to interpret this new metric, it is convenient to define polar coordinates in the parameter space

$$
\rho=\sqrt{\delta^{2}+\gamma^{2}}, \quad \tan \zeta=\frac{\delta}{\gamma} .
$$

Performing a charge redefinition and a change of coordinates

$$
\begin{aligned}
\left(\begin{array}{l}
M^{\prime} \\
N^{\prime}
\end{array}\right) & =\left(\begin{array}{cc}
\cos 2 \zeta & -\sin 2 \zeta \\
\sin 2 \zeta & \cos 2 \zeta
\end{array}\right)\left(\begin{array}{l}
\rho M \\
\rho N
\end{array}\right), \\
t^{\prime}=\frac{t}{\rho}, \quad r^{\prime} & =\rho r+M^{\prime}(1-\cos 2 \zeta)-N^{\prime} \sin 2 \zeta,
\end{aligned}
$$

the metric simplifies to

$$
\begin{aligned}
d s^{\prime 2} & =-f\left(r^{\prime}\right)\left(d t^{\prime}-2 N^{\prime} \cos \theta d \phi\right)^{2}+\frac{d r^{\prime 2}}{f\left(r^{\prime}\right)}+\left(r^{\prime 2}+N^{\prime 2}\right) d \Omega_{2}^{2}, \\
f\left(r^{\prime}\right) & =\frac{r^{\prime 2}-2 M^{\prime} r^{\prime}-N^{\prime 2}}{r^{\prime 2}+N^{\prime 2}} .
\end{aligned}
$$

Hence, it is still a member of the Taub-NUT family. The self-dual part of $F_{\mu \nu}$ transforms as (5.5). The integrated Ernst one-form transforms as

$$
\sigma^{\prime}=\frac{1}{\delta^{2}+\gamma^{2}} \frac{\delta \sigma+i \gamma}{i \gamma \sigma+\delta}
$$

After the transformation, (5.12) also holds with

$$
c^{\prime}=\frac{1}{\gamma^{2}+\delta^{2}} \quad A^{\prime}=-\frac{(\delta+i \gamma)^{4}}{4(M-i N)},
$$

in agreement with (54) in [73].

Let us now study the implications for the single copy. The single copy of (2.20) can be written in flat spherical coordinates $(\tilde{t}, \tilde{r}, \theta, \phi)(2.26)$

$$
F_{T}=-\frac{M}{\tilde{r}^{2}} d \tilde{t} \wedge d \tilde{r}-N \sin \theta d \theta \wedge d \phi .
$$

Hence, the single copy of the transformed space-time, on the same background reads

$$
F_{T}^{\prime}=-\frac{M^{\prime}}{\tilde{r}^{2}} d \tilde{t} \wedge d \tilde{r}-N^{\prime} \sin \theta d \theta \wedge d \phi .
$$


Using (5.15), it can be checked that the transformation in terms of the Ehlers group parameters is

$$
F_{T}^{\prime}=\rho \cos (2 \zeta) F_{T}+\rho \sin (2 \zeta) \tilde{F}_{T} .
$$

This corresponds to an electromagnetic duality rotation and a rescaling by $\rho$. Both transformations are contained in the electromagnetic duality, where the rescaling can be interpreted as the transformation of the gauge coupling [63]. The zeroth copy is affected similarly, transforming using $M \rightarrow M^{\prime}, N \rightarrow N^{\prime}$, leaving the double copy structure intact. The Weyl double copy

$$
C_{\mu \nu \rho \sigma}^{+}=\frac{2}{\sigma^{+}} C_{\mu \nu \rho \sigma}\left[F^{+}\right]
$$

where $F^{\prime}+$ is the self-dual part of the transformed single-copy Killing tensor and $\sigma^{+}=$ $\sigma-c=-2(N+i M) /(N+i r)$, is preserved: (5.22) transforms directly to the double copy in the transformed spacetime

$$
C_{\mu \nu \rho \sigma}^{\prime+}=\frac{2}{\sigma^{+^{\prime}}} C_{\mu \nu \rho \sigma}\left[F^{\prime+}\right]
$$

where $F^{+}$is the self-dual part of the single-copy Killing tensor, now defined using the shifted metric (and the same Killing vector, although note of course that the co-vector differs in the new spacetime), and the transformed Ernst scalar is

$$
\sigma^{\prime}=-\frac{2(M-i N)}{(\gamma-i \delta)(2 \gamma M+N(\delta-i \gamma)+r(i \delta-\gamma))} .
$$

We see that in terms of the action on the fields, a restricted set of the $\operatorname{SL}(2, \mathbb{R})$ transformations act in this case, and the orbit is within the Taub-NUT class of metrics. The two degrees of freedom are realised by the rotation parameter $\zeta$ and scaling $\rho$.

\subsection{More general type $\mathrm{D}$ metrics}

Now we may consider the general class of metrics given in (4.17). One has the Killing vectors $U=(1,0,0,0)$ and $V=(0,1,0,0)$. Defining the Killing forms $F_{\mu \nu}^{U}=2 \partial_{[\mu} U_{\nu]}, F_{\mu \nu}^{V}=2 \partial_{[\mu} V_{\nu]}$, we find that these are simply related to the single copy Maxwell tensor (4.12) by

$$
F_{T}=\frac{1}{2}\left(F^{U}+i \tilde{F}^{V}\right)
$$

which is equivalent to $F_{T}^{ \pm}=\frac{1}{2} F^{U \pm i V}$. The double copy is then a formula of the form of the vanishing of a Mars-Simons tensor:

$$
C_{\mu \nu \rho \sigma}^{+}=\left.\frac{1}{\sigma^{U+i V}} C_{\mu \nu \rho \sigma}\left[F^{(U+i V)+}\right]\right|_{e \rightarrow m, g \rightarrow n},
$$

with the Ernst scalar given by

$$
\sigma^{U+i V}=-\frac{i(1-p q)(m-i n)}{p+i q} .
$$


The metric (4.17) is complex, as is the relevant Killing vector $U+i V$. The derivation of the equations (5.3) relies on a real Killing vector and so cannot be used directly in this case. Thus consider instead the real form of the metric

$$
d s^{2}=\frac{1}{(1-y r)^{2}}\left[-\frac{\Delta_{r}}{\Sigma}\left(d t+y^{2} d \psi\right)^{2}+\frac{\Delta_{y}}{\Sigma}\left(d t-r^{2} d \psi\right)^{2}+\frac{\Sigma}{\Delta_{r}} d r^{2}+\frac{\Sigma}{\Delta_{y}} d y^{2}\right],
$$

with $\Sigma=r^{2}+y^{2}$ and

$$
\begin{aligned}
& \Delta_{r}=k\left(1-r^{4}\right)-2 m r+\epsilon r^{2}-2 n r^{3}, \\
& \Delta_{y}=k\left(1-y^{4}\right)+2 n y-\epsilon y^{2}+2 m y^{3} .
\end{aligned}
$$

Defining the single-copy Maxwell tensor

$$
F=\frac{1}{\left(r^{2}+y^{2}\right)^{2}}\left[\left(2 n r y-m\left(y^{2}-r^{2}\right)\right)\left(d t+y^{2} d \psi\right) d r+\left(2 m r y+n\left(y^{2}-r^{2}\right)\right)\left(d t-r^{2} d \psi\right) d y\right]
$$

then the Weyl double copy is given by

$$
C_{\mu \nu \rho \sigma}^{+}=\frac{16(i y-r)}{(m-i n)(1-r y)} C_{\mu \nu \rho \sigma}\left[F^{+}\right]
$$

and its conjugate.

Now consider the Killing vector $K=\partial_{t}$. The solution of the equations (5.3) here is more involved: these are solved by $W=\left(W_{t}, 0,0, W_{\psi}\right)-\left(1-\delta^{2}, 0,0,0\right)$ with

$$
\begin{aligned}
W_{t}= & \frac{\gamma}{(r y-1)^{4}\left(r^{2}+y^{2}\right)}\left[4 \gamma m^{2}\left(y^{4}+1\right)+4 \gamma n^{2}\left(r^{4}+1\right)\right. \\
& +4 n\left(-\gamma e\left(r^{3}+y\right)+\gamma k\left(r^{5}+r^{3} y^{2}-3 r^{2} y+2 r-y^{3}\right)+\delta r(r y-1)^{3}\right) \\
& +\left(\left(r^{2}+y^{2}\right)\left(\gamma e^{2}+2 \gamma e k\left(y^{2}-r^{2}\right)+\gamma k^{2}\left(r^{4}+2 r^{2} y^{2}-8 r y+y^{4}+4\right)+4 k \delta(r y-1)^{3}\right)\right. \\
& -4 m\left(\gamma e\left(r+y^{3}\right)-\gamma k r^{3}+\gamma k r^{2} y^{3}-3 \gamma k r y^{2}+\gamma k y^{5}+2 \gamma k y-2 \gamma n\left(r^{2}+y^{2}\right)\right. \\
& \left.+\delta r^{3} y^{4}-3 \delta r^{2} y^{3}+3 \delta r y^{2}-\delta y\right)
\end{aligned}
$$

and

$$
\begin{aligned}
W_{\psi}= & \frac{2 \gamma}{(r y-1)^{3}\left(r^{2}+y^{2}\right)}\left(2 \gamma k^{2}\left(r^{4}-y^{4}\right)-2 \gamma m^{2} r y^{3}-2 \gamma m^{2} y^{2}\right. \\
& +k\left(-2 \gamma e r y\left(r^{2}+y^{2}\right)+2 \gamma m y^{2}\left(r^{3}+r y^{2}+2 y\right)+\delta\left(r^{5} y-r^{4}-r y^{5}+y^{4}\right)\right) \\
& +2 n r\left(\gamma(-e) r y+\gamma k r\left(r^{2} y+2 r+y^{3}\right)+\delta y\left(r^{4} y-r^{3}+2 r^{2} y^{3}-3 r y^{2}+y\right)\right) \\
& +2 \gamma e m r y^{2}-2 \delta e r^{4} y^{2}+3 \delta e r^{3} y-\delta e r^{2}-2 \delta e r^{2} y^{4}+3 \delta e r y^{3}-\delta e y^{2} \\
& \left.+4 \delta m r^{4} y^{3}-6 \delta m r^{3} y^{2}+2 \delta m r^{2} y^{5}+2 \delta m r^{2} y-2 \delta m r y^{4}+2 \gamma n^{2} r^{2}(r y+1)\right),
\end{aligned}
$$

with the Killing tensor here $F_{\mu \nu}=2 \partial_{[\mu} K_{\nu]}$ and

$$
\sigma=\frac{1}{(r-i y)(1-r y)^{2}}\left((r-i y)\left(e+k\left(-r^{2}-2 i r y+y^{2}+2 i\right)\right)+2 i m\left(y^{2}+i\right)-2 n\left(r^{2}-i\right)\right) .
$$


The shifted Maxwell tensor is given by (5.5) and the new metric by (5.1) with $W$ and $\sigma$ given by the expressions above. Whilst this will lead to an $\operatorname{SL}(2, \mathbb{R})$ action on the original solution, we note that the single-copy Maxwell tensor is not equal to the Killing tensor obtained from the Killing vector $K=\partial_{t}$. It would appear from this that one can either have the double copy arising from a complex Killing vector but without the $\mathrm{SL}(2, \mathbb{R})$ action as described in [73], or can keep the group action based on a real Killing vector but without the double copy being preserved. We have seen above that this issue does not arise for the Taub-NUT solution, which is a certain limit of the metric (4.17); in addition, some more recent work has studied complex Killing vectors and their Mars-Simon tensors [76, 77]. It may thus be worth exploring this case further.

\subsection{The Eguchi-Hanson metric}

It is of interest to consider a Riemannian metric example and we turn to the Eguchi-Hanson metric

$$
d s^{2}=2 d u d v-2 d X d Y+\frac{\lambda}{(u v-X Y)^{3}}(v d u-X d Y)^{2},
$$

with coordinates $(u, v, X, Y)$ and constant $\lambda$. The single-copy (self-dual) Maxwell tensor is $[46,59]$

$$
F=\frac{2 \lambda}{(u v-X Y)^{3}}((u v+X Y)(d u \wedge d v-d X \wedge d Y)-2 v Y d u \wedge d X+2 u X d v \wedge d Y) .
$$

Consider the Killing vector

$$
K^{\mu}=(u,-v,-X, Y)
$$

and Killing two-form

$$
K_{\mu \nu}=2 \partial_{[\mu} K_{\nu]}
$$

The single-copy Maxwell tensor is then given by

$$
F_{\mu \nu}=K_{\mu \nu}^{+}
$$

We have the relations

$$
\sigma_{\mu}^{+}:=2 K^{\nu} K_{\nu \mu}^{+}=\partial_{\mu} \sigma^{+}, \quad \sigma_{\mu}^{-}:=K^{\nu} K_{\nu \mu}^{-}=\partial_{\mu} \sigma^{-},
$$

with

$$
\sigma^{+}=-\frac{2 \lambda}{(u v-X Y)}, \quad \sigma^{-}=4(u v-X Y) .
$$

We now consider the equations (5.3) with $\sigma \rightarrow \sigma^{+}, \bar{\sigma} \rightarrow \sigma^{-}$and $\xi$ the Killing vector (5.37). These are solved by

$$
\begin{aligned}
W_{\mu}= & -\left(\frac{8 \lambda \gamma^{2}}{u v-X Y}+2 i \gamma \delta\right)(v,-u, Y,-X)-\frac{2 i \lambda \gamma \delta}{(u v-X Y)^{2}}(v, 0,0,-X) \\
& -\left(1-8 \lambda \gamma^{2}-\delta^{2}\right)\left(\frac{1}{u}, 0,0,0\right) .
\end{aligned}
$$


The new metric is given by (5.1) with $W$ given by the expression above. This is a complicated expression which we will not reproduce here. We have checked that this is Ricci-flat. The single-copy Maxwell tensor $K_{\mu \nu}^{+}$transforms in the same way as $F^{+}$in (5.5), and the transformation of its dual is the conjugate of this. It can be checked that the transformed tensors are (anti-)self-dual with respect to the transformed metric (5.1), and agree with the new Killing two form obtained from (5.38) using the same Killing vector (5.37) but with the index lowered with the new metric.

Considering the Weyl tensor, we have the double copy relation for the Eguchi-Hanson metric

$$
C_{\mu \nu \rho \sigma}^{E H}=-\frac{u v-X Y}{\lambda} C_{\mu \nu \rho \sigma}\left[K^{+}\right]
$$

where $K^{+}$is the single copy Maxwell tensor (5.39). Note that the Weyl tensor for the Eguchi-Hanson metric is self-dual. We find that the equivalents of (5.41) in the transformed metric are

$$
\sigma^{\prime}=-\frac{2 i \lambda}{\left(2 \gamma \delta \lambda+i \delta^{2}(u v-X Y)\right)}, \quad \sigma^{\prime}=\frac{1}{\left(4 \gamma^{2}(u v-X Y)+i \gamma \delta\right)},
$$

and that the double copy relationship is preserved by a general transformation, with

$$
C_{\mu \nu \rho \sigma}^{\prime \pm}=\frac{2}{\sigma^{\prime \pm}} C_{\mu \nu \rho \sigma}\left[K^{\prime \pm}\right]
$$

To gain some insight into the action of $\mathrm{SL}(2, \mathbb{R})$ in this example, consider the transformations with $\delta=0$. The shifted Maxwell fields in this case are given by

$$
K^{\prime}=\frac{1}{2 \lambda \gamma^{2}}\left(-d u \wedge d v+\frac{\left(1-8 \lambda \gamma^{2}\right)}{u} d u \wedge d(X Y)\right)-4 d X \wedge d Y
$$

and

$$
\begin{aligned}
K^{\prime}-= & \frac{-u v+X Y\left(1-16 \lambda \gamma^{2}\right)}{4(u v-X Y)^{3} \gamma^{2}} d u \wedge d v+\frac{Y\left(u v-X Y+8 \lambda \gamma^{2}(u v+X Y)\right)}{4 u(u v-X Y)^{3} \gamma^{2}} d u \wedge d X \\
& +\frac{X\left(1-8 \lambda \gamma^{2}\right)}{4 u(u v-X Y)^{2} \gamma^{2}} d u \wedge d Y-\frac{4 u \lambda X}{(u v-X Y)^{3}} d v \wedge d Y+\frac{2 \lambda(u v+X Y)}{(u v-X Y)^{3}} d X \wedge d Y .
\end{aligned}
$$

For the transformed metric in this case, from (5.45) the new Weyl tensor is now anti-selfdual and obeys the double copy relationship $C_{\mu \nu \rho \sigma}^{\prime-}=8 \gamma^{2}(u v-X Y) C_{\mu \nu \rho \sigma}\left[K^{-^{\prime}}\right]$, where $K_{\mu \nu}^{-^{\prime}}$ is the shifted Maxwell tensor given in (5.47). When the parameter $\gamma$ satisfies $8 \lambda \gamma^{2}=1$ we find that $K_{\mu \nu}^{\prime}=-K_{\mu \nu}^{+}$(note that the dual of a tensor is defined with respect to different metrics on the two sides of this equation). Thus the roles of the electromagnetic fields $E$ and $B$ are exchanged by this transformation. The new metric is given simply in terms of the original Eguchi-Hanson metric by the interchange of $(u, v) \leftrightarrow(X, Y)$. The double copy relations are consistent with this. It would be interesting to follow up with a full study of the action of the transformations when $\delta \neq 0$. 


\subsection{Higher dimensions}

Higher-dimensional double copies can be studied via a direct application of the formula (4.4), but it should be kept in mind that in many cases of interest there is more structure in higher-dimensions that may play a key role in consideration of double copies. The review [78], for example, summarises work on symmetries of higher-dimensional KerrNUT-(A)dS black hole spacetimes. These symmetries are linked with the presence of Killing and Killing-Yano tensors, which feature strongly in the discussion of the special properties of these spacetimes, such as their algebraic type, the integrability of geodesic motion, and separability of the Hamilton-Jacobi, Klein-Gordon, and Dirac equations. The fundamental Killing object in these discussions is the "principal tensor" $h$, which generates a complete set of explicit and hidden symmetries and uniquely determines the geometry of the Kerr-NUT-(A)dS metric.

The principal tensor, and those other tensors generated by it, might be expected to also play a role in defining tensor double copies for such spacetimes in higher dimensions. If so, we would expect a relationship between any Maxwell tensor (or other two forms to be used in the double copy) and the principal tensor and its descendants. This might first be investigated in four dimensions. In the four-dimensional spacetime (4.17) discussed above, with the two null co-vectors

$$
\begin{aligned}
K & =d u+q^{2} d v, \\
L & =d u-p^{2} d v,
\end{aligned}
$$

the Maxwell single copy gauge field is given by

$$
A=\frac{1}{p^{2}+q^{2}}(g p K+e q L) .
$$

Its field strength is given by (4.12) above, i.e.

$$
F=\frac{1}{\left(p^{2}+q^{2}\right)^{2}}(\alpha d p K+\beta d q L)
$$

with $\alpha=-2 e p q-e\left(p^{2}-q^{2}\right), \beta=-2 g p q+e\left(p^{2}-q^{2}\right)$. The principal tensor $h$ and its dual for this case are given by [78]

$$
\begin{aligned}
& h=\frac{1}{(1-p q)^{3}}(p d p K-q d q L), \\
& \tilde{h}=\frac{1}{(1-p q)^{3}}(q d p K+p d q L) .
\end{aligned}
$$

One can check that the Maxwell single copy (5.50) can be expressed in terms of the principal tensor as

$$
F=a h+b \tilde{h},
$$

where $a=\Omega^{3}\left(-3 p q(g p+e q)+g q^{3}+e p^{3}\right)$ and $b=\Omega^{3}\left(-3 p q(e p-g q)+e q^{3}-g p^{3}\right)$, with $\Omega=\frac{1-p q}{p^{2}+q^{2}}$. Curiously, the factors in the coefficients $a, b$ (with $e \rightarrow m, g \rightarrow n$ ) also appear 
in some of the components of the Weyl tensor. The result (5.52) implies that this fourdimensional Weyl double copy can be written purely in terms of the principal tensor. It would be interesting to explore higher-dimensional Weyl double copies by seeing how the principal tensor and its descendants, the related Killing tensors, along with the Maxwell tensor where this is independent, might be used in formulæ like (4.4) to map to the Weyl tensor; spinor analogues might also be explored of course.

\section{Discussion}

We have explored the double copy formalism in the context of solution generating symmetries in relativity, and in particular how these symmetries in general relativity are related to hidden or duality symmetries in the single copy gauge theory. We have used two complementary techniques, the Kerr-Schild and the Weyl double copy formalisms, and have seen in a number of cases that the transformations in the gauge theory and the corresponding transformations on the gravity side are such that the double copy structure is preserved. This seems to us further evidence that the double copy is beyond just a perturbative symmetry for amplitudes but a fascinating relation between gravity and gauge theory.

\section{Acknowledgments}

DSB and WJS are supported by the U.K. Science and Technology Facilities Council (STFC) with consolidated grant ST/L000415/1, String Theory, Gauge Theory and Duality. DPV is supported by a Royal Society studentship and RA is supported by a student scholarship from the Ministry of Higher Education of the UAE. We thank Joe Hayling, Ricardo Monteiro and Chris White for discussions on this topic.

Open Access. This article is distributed under the terms of the Creative Commons Attribution License (CC-BY 4.0), which permits any use, distribution and reproduction in any medium, provided the original author(s) and source are credited.

\section{References}

[1] Z. Bern, J.J.M. Carrasco and H. Johansson, New Relations for Gauge-Theory Amplitudes, Phys. Rev. D 78 (2008) 085011 [arXiv: 0805.3993] [inSPIRE].

[2] Z. Bern, J.J.M. Carrasco and H. Johansson, Perturbative Quantum Gravity as a Double Copy of Gauge Theory, Phys. Rev. Lett. 105 (2010) 061602 [arXiv:1004.0476] [INSPIRE].

[3] Z. Bern, T. Dennen, Y.-t. Huang and M. Kiermaier, Gravity as the Square of Gauge Theory, Phys. Rev. D 82 (2010) 065003 [arXiv: 1004.0693] [InSPIRE].

[4] N.E.J. Bjerrum-Bohr, P.H. Damgaard and P. Vanhove, Minimal Basis for Gauge Theory Amplitudes, Phys. Rev. Lett. 103 (2009) 161602 [arXiv:0907.1425] [INSPIRE].

[5] S. Stieberger, Open and Closed vs. Pure Open String Disk Amplitudes, arXiv:0907.2211 [INSPIRE]. 
[6] N.E.J. Bjerrum-Bohr, P.H. Damgaard, T. Sondergaard and P. Vanhove, Monodromy and Jacobi-like Relations for Color-Ordered Amplitudes, JHEP 06 (2010) 003 [arXiv: 1003.2403] [INSPIRE].

[7] B. Feng, R. Huang and Y. Jia, Gauge Amplitude Identities by On-shell Recursion Relation in S-matrix Program, Phys. Lett. B 695 (2011) 350 [arXiv:1004.3417] [INSPIRE].

[8] S.H. Henry Tye and Y. Zhang, Dual Identities inside the Gluon and the Graviton Scattering Amplitudes, JHEP 06 (2010) 071 [Erratum ibid. 1104 (2011) 114] [arXiv:1003.1732] [INSPIRE].

[9] C.R. Mafra, O. Schlotterer and S. Stieberger, Explicit BCJ Numerators from Pure Spinors, JHEP 07 (2011) 092 [arXiv: 1104.5224] [INSPIRE].

[10] R. Monteiro and D. O'Connell, The Kinematic Algebra From the Self-Dual Sector, JHEP 07 (2011) 007 [arXiv:1105.2565] [INSPIRE].

[11] N.E.J. Bjerrum-Bohr, P.H. Damgaard, R. Monteiro and D. O'Connell, Algebras for Amplitudes, JHEP 06 (2012) 061 [arXiv:1203.0944] [INSPIRE].

[12] H. Kawai, D.C. Lewellen and S.H.H. Tye, A Relation Between Tree Amplitudes of Closed and Open Strings, Nucl. Phys. B 269 (1986) 1 [INSPIRE].

[13] Z. Bern, L.J. Dixon, D.C. Dunbar, M. Perelstein and J.S. Rozowsky, On the relationship between Yang-Mills theory and gravity and its implication for ultraviolet divergences, Nucl. Phys. B 530 (1998) 401 [hep-th/9802162] [INSPIRE].

[14] M.B. Green, J.H. Schwarz and L. Brink, $N=4$ Yang-Mills and $N=8$ Supergravity as Limits of String Theories, Nucl. Phys. B 198 (1982) 474 [INSPIRE].

[15] Z. Bern, J.S. Rozowsky and B. Yan, Two loop four gluon amplitudes in $N=4$ superYang-Mills, Phys. Lett. B 401 (1997) 273 [hep-ph/9702424] [INSPIRE].

[16] J.J.M. Carrasco and H. Johansson, Five-Point Amplitudes in $N=4$ Super-Yang-Mills Theory and $N=8$ Supergravity, Phys. Rev. D 85 (2012) 025006 [arXiv:1106.4711] [INSPIRE].

[17] J.J.M. Carrasco, M. Chiodaroli, M. Günaydin and R. Roiban, One-loop four-point amplitudes in pure and matter-coupled $N \leq 4$ supergravity, JHEP 03 (2013) 056 [arXiv:1212.1146] [INSPIRE].

[18] C.R. Mafra and O. Schlotterer, The Structure of n-Point One-Loop Open Superstring Amplitudes, JHEP 08 (2014) 099 [arXiv:1203.6215] [INSPIRE].

[19] R.H. Boels, R.S. Isermann, R. Monteiro and D. O'Connell, Colour-Kinematics Duality for One-Loop Rational Amplitudes, JHEP 04 (2013) 107 [arXiv:1301.4165] [INSPIRE].

[20] N.E.J. Bjerrum-Bohr, T. Dennen, R. Monteiro and D. O'Connell, Integrand Oxidation and One-Loop Colour-Dual Numerators in N=4 Gauge Theory, JHEP 07 (2013) 092 [arXiv: 1303.2913] [INSPIRE].

[21] Z. Bern, S. Davies, T. Dennen, Y.-t. Huang and J. Nohle, Color-Kinematics Duality for Pure Yang-Mills and Gravity at One and Two Loops, Phys. Rev. D 92 (2015) 045041 [arXiv: 1303.6605] [INSPIRE].

[22] Z. Bern, S. Davies and T. Dennen, The Ultraviolet Structure of Half-Maximal Supergravity with Matter Multiplets at Two and Three Loops, Phys. Rev. D 88 (2013) 065007 [arXiv:1305.4876] [INSPIRE]. 
[23] J. Nohle, Color-Kinematics Duality in One-Loop Four-Gluon Amplitudes with Matter, Phys. Rev. D 90 (2014) 025020 [arXiv: 1309.7416] [InSPIRE].

[24] Z. Bern, S. Davies, T. Dennen, A.V. Smirnov and V.A. Smirnov, Ultraviolet Properties of $N=4$ Supergravity at Four Loops, Phys. Rev. Lett. 111 (2013) 231302 [arXiv:1309.2498] [INSPIRE].

[25] S.G. Naculich, H. Nastase and H.J. Schnitzer, All-loop infrared-divergent behavior of most-subleading-color gauge-theory amplitudes, JHEP 04 (2013) 114 [arXiv:1301.2234] [INSPIRE].

[26] Y.-J. Du, B. Feng and C.-H. Fu, Dual-color decompositions at one-loop level in Yang-Mills theory, JHEP 06 (2014) 157 [arXiv:1402.6805] [INSPIRE].

[27] C.R. Mafra and O. Schlotterer, Towards one-loop SYM amplitudes from the pure spinor BRST cohomology, Fortsch. Phys. 63 (2015) 105 [arXiv:1410.0668] [INSPIRE].

[28] Z. Bern, S. Davies and T. Dennen, Enhanced ultraviolet cancellations in $\mathcal{N}=5$ supergravity at four loops, Phys. Rev. D 90 (2014) 105011 [arXiv:1409.3089] [INSPIRE].

[29] C.R. Mafra and O. Schlotterer, Two-loop five-point amplitudes of super Yang-Mills and supergravity in pure spinor superspace, JHEP 10 (2015) 124 [arXiv:1505.02746] [INSPIRE].

[30] S. He, R. Monteiro and O. Schlotterer, String-inspired BCJ numerators for one-loop MHV amplitudes, JHEP 01 (2016) 171 [arXiv:1507.06288] [INSPIRE].

[31] Z. Bern, S. Davies and J. Nohle, Double-Copy Constructions and Unitarity Cuts, Phys. Rev. D 93 (2016) 105015 [arXiv:1510.03448] [InSPIRE].

[32] G. Mogull and D. O'Connell, Overcoming Obstacles to Colour-Kinematics Duality at Two Loops, JHEP 12 (2015) 135 [arXiv:1511.06652] [INSPIRE].

[33] M. Chiodaroli, M. Günaydin, H. Johansson and R. Roiban, Spontaneously Broken Yang-Mills-Einstein Supergravities as Double Copies, JHEP 06 (2017) 064 [arXiv: 1511.01740] [INSPIRE].

[34] Z. Bern, J.J.M. Carrasco, W.-M. Chen, H. Johansson, R. Roiban and M. Zeng, Five-loop four-point integrand of $N=8$ supergravity as a generalized double copy, Phys. Rev. D 96 (2017) 126012 [arXiv:1708.06807] [INSPIRE].

[35] H. Johansson and A. Ochirov, Color-Kinematics Duality for QCD Amplitudes, JHEP 01 (2016) 170 [arXiv: 1507.00332] [INSPIRE].

[36] S. Oxburgh and C.D. White, BCJ duality and the double copy in the soft limit, JHEP 02 (2013) 127 [arXiv:1210.1110] [INSPIRE].

[37] C.D. White, Factorization Properties of Soft Graviton Amplitudes, JHEP 05 (2011) 060 [arXiv:1103.2981] [INSPIRE].

[38] S. Melville, S.G. Naculich, H.J. Schnitzer and C.D. White, Wilson line approach to gravity in the high energy limit, Phys. Rev. D 89 (2014) 025009 [arXiv:1306.6019] [InSPIRE].

[39] A. Luna, S. Melville, S.G. Naculich and C.D. White, Next-to-soft corrections to high energy scattering in QCD and gravity, JHEP 01 (2017) 052 [arXiv:1611.02172] [INSPIRE].

[40] R. Saotome and R. Akhoury, Relationship Between Gravity and Gauge Scattering in the High Energy Limit, JHEP 01 (2013) 123 [arXiv:1210.8111] [INSPIRE].

[41] A. Sabio Vera, E. Serna Campillo and M.A. Vazquez-Mozo, Color-Kinematics Duality and the Regge Limit of Inelastic Amplitudes, JHEP 04 (2013) 086 [arXiv:1212.5103] [INSPIRE]. 
[42] H. Johansson, A. Sabio Vera, E. Serna Campillo and M.Á. Vázquez-Mozo, Color-Kinematics Duality in Multi-Regge Kinematics and Dimensional Reduction, JHEP 10 (2013) 215 [arXiv: 1307.3106] [INSPIRE].

[43] H. Johansson, A. Sabio Vera, E. Serna Campillo and M.Á. Vazquez-Mozo, Color-kinematics duality and dimensional reduction for graviton emission in Regge limit, in International Workshop on Low X Physics (Israel 2013), Eilat Israel (2013) [arXiv:1310.1680] [INSPIRE].

[44] R. Monteiro, D. O'Connell and C.D. White, Black holes and the double copy, JHEP 12 (2014) 056 [arXiv: 1410.0239] [INSPIRE].

[45] A. Luna, R. Monteiro, D. O'Connell and C.D. White, The classical double copy for Taub-NUT spacetime, Phys. Lett. B 750 (2015) 272 [arXiv: 1507.01869] [INSPIRE].

[46] D.S. Berman, E. Chacón, A. Luna and C.D. White, The self-dual classical double copy and the Eguchi-Hanson instanton, JHEP 01 (2019) 107 [arXiv: 1809. 04063] [INSPIRE].

[47] S. Sabharwal and J.W. Dalhuisen, Anti-Self-Dual Spacetimes, Gravitational Instantons and Knotted Zeros of the Weyl Tensor, JHEP 07 (2019) 004 [arXiv: 1904.06030] [INSPIRE].

[48] A. Anastasiou, L. Borsten, M.J. Duff, L.J. Hughes and S. Nagy, Yang-Mills origin of gravitational symmetries, Phys. Rev. Lett. 113 (2014) 231606 [arXiv:1408.4434] [INSPIRE].

[49] L. Borsten and M.J. Duff, Gravity as the square of Yang-Mills?, Phys. Scripta 90 (2015) 108012 [arXiv: 1602 .08267] [INSPIRE].

[50] A. Anastasiou et al., Twin supergravities from Yang-Mills theory squared, Phys. Rev. D 96 (2017) 026013 [arXiv:1610.07192] [InSPIRE].

[51] A. Anastasiou, L. Borsten, M.J. Duff, A. Marrani, S. Nagy and M. Zoccali, Are all supergravity theories Yang-Mills squared?, Nucl. Phys. B 934 (2018) 606 [arXiv: 1707.03234] [INSPIRE].

[52] G.L. Cardoso, S. Nagy and S. Nampuri, A double copy for $\mathcal{N}=2$ supergravity: a linearised tale told on-shell, JHEP 10 (2016) 127 [arXiv:1609.05022] [INSPIRE].

[53] L. Borsten, $D=6, \mathcal{N}=(2,0)$ and $\mathcal{N}=(4,0)$ theories, Phys. Rev. D 97 (2018) 066014 [arXiv: 1708.02573] [INSPIRE].

[54] A. Anastasiou, L. Borsten, M.J. Duff, A. Marrani, S. Nagy and M. Zoccali, The Mile High Magic Pyramid, arXiv:1711.08476 [InSPIRE].

[55] A. Anastasiou, L. Borsten, M.J. Duff, S. Nagy and M. Zoccali, Gravity as Gauge Theory Squared: A Ghost Story, Phys. Rev. Lett. 121 (2018) 211601 [arXiv:1807.02486] [INSPIRE].

[56] G. Lopes Cardoso, G. Inverso, S. Nagy and S. Nampuri, Comments on the double copy construction for gravitational theories, PoS (CORFU2017) 177 (2018) [arXiv: 1803.07670] [INSPIRE].

[57] C. Montonen and D.I. Olive, Magnetic Monopoles as Gauge Particles?, Phys. Lett. B 72 (1977) 117 [INSPIRE].

[58] H.A. Buchdahl, Reciprocal Static Metrics and Scalar Fields in the General Theory of Relativity, Phys. Rev. 115 (1959) 1325 [inSPIRE].

[59] A. Luna, R. Monteiro, I. Nicholson and D. O'Connell, Type D Spacetimes and the Weyl Double Copy, Class. Quant. Grav. 36 (2019) 065003 [arXiv:1810.08183] [InSPIRE].

[60] Y.-T. Huang, U. Kol and D. O'Connell, The Double Copy of Electric-Magnetic Duality, arXiv:1911.06318 [INSPIRE]. 
[61] A.H. Taub, Empty space-times admitting a three parameter group of motions, Annals Math. 53 (1951) 472 [INSPIRE].

[62] E. Newman, L. Tamburino and T. Unti, Empty space generalization of the Schwarzschild metric, J. Math. Phys. 4 (1963) 915 [inSPIRE].

[63] T. Ortin, Gravity and Strings, Cambridge Monographs on Mathematical Physics, Cambridge University Press, Cambridge U.K. (2015).

[64] J.F. Plebañski, A class of solutions of Einstein-Maxwell equations, Annals Phys. 90 (1975) 196 [INSPIRE].

[65] Z.W. Chong, G.W. Gibbons, H. Lü and C.N. Pope, Separability and Killing tensors in Kerr-Taub-NUT-de Sitter metrics in higher dimensions, Phys. Lett. B 609 (2005) 124 [hep-th/0405061] [INSPIRE].

[66] F.J. Ernst, New formulation of the axially symmetric gravitational field problem, Phys. Rev. 167 (1968) 1175 [InSPIRE].

[67] J. Ehlers, Transformations of static exterior solutions of Einstein's gravitational field equations into different solutions by means of conformal mapping, Colloq. Int. CNRS $\mathbf{9 1}$ (1962) 275 [INSPIRE].

[68] D. Momeni, M. Nouri-Zonoz and R. Ramezani-Arani, MM-NUT disk space via Ehlers transformation, Phys. Rev. D 72 (2005) 064023 [gr-qc/0508036] [INSPIRE].

[69] R.P. Geroch, A Method for generating solutions of Einstein's equations, J. Math. Phys. 12 (1971) 918 [INSPIRE].

[70] R. Monteiro, I. Nicholson and D. O'Connell, Spinor-helicity and the algebraic classification of higher-dimensional spacetimes, Class. Quant. Grav. 36 (2019) 065006 [arXiv:1809.03906] [INSPIRE].

[71] J.F. Plebanski and M. Demianski, Rotating, charged and uniformly accelerating mass in general relativity, Annals Phys. 98 (1976) 98 [INSPIRE].

[72] J.B. Griffiths and J. Podolsky, A New look at the Plebanski-Demianski family of solutions, Int. J. Mod. Phys. D 15 (2006) 335 [gr-qc/0511091] [InSPIRE].

[73] M. Mars, Space-time Ehlers group: Transformation law for the Weyl tensor, Class. Quant. Grav. 18 (2001) 719 [gr-qc/0101020] [INSPIRE].

[74] M. Mars, T.-T. Paetz and J.M.M. Senovilla, Classification of Kerr-de Sitter-like spacetimes with conformally flat I, Class. Quant. Grav. 34 (2017) 095010 [arXiv:1610.09846] [INSPIRE].

[75] A. G.-P. Gómez-Lobo, Vacuum type D initial data, Class. Quant. Grav. 33 (2016) 175005 [Erratum ibid. 35 (2018) 079501] [arXiv: 1602.08075] [INSPIRE].

[76] T.-T. Paetz, Algorithmic characterization results for the Kerr-NUT-(A)dS space-time. I. A space-time approach, J. Math. Phys. 58 (2017) 042501 [arXiv:1701.02959] [InSPIRE].

[77] T.-T. Paetz, Algorithmic characterization results for the Kerr-NUT-(A)dS space-time. II. KIDs for the Kerr-(A)(de Sitter) family, J. Math. Phys. 58 (2017) 042502 [arXiv: 1701.03315] [INSPIRE].

[78] V. Frolov, P. Krtous and D. Kubiznak, Black holes, hidden symmetries and complete integrability, Living Rev. Rel. 20 (2017) 6 [arXiv:1705.05482] [INSPIRE]. 